\title{
A Comparative Study of Two Disposable Supraglottic Devices in Diagnostic Laparoscopy in Gynecology
}

\author{
Suman Chattopadhyay, Sebanti Goswami
}

\begin{abstract}
Introduction: This prospective study compares the efficacy of two disposable supraglottic airway devices with separate gastric access and integral bite block, the inflatable cuff of the LMA Supreme ${ }^{\mathrm{TM}}$ against the noninflatable $\mathrm{i}-\mathrm{gel}^{\mathrm{TM}}$ cuff in providing an adequate seal for laparoscopic surgery.
\end{abstract}

\begin{abstract}
Materials and methods: Ninety women of ASA grade I and II undergoing diagnostic laparoscopy with Trendelenburg position were randomly allocated to receive either size 3 LMA Supreme or i-gel. Induction and maintenance protocols were similar and patients were not paralyzed. We compared the airway leak pressure, ease and time to insert the device and a $10 \mathrm{FG}$ nasogastric tube through it, and to note complications if any.
\end{abstract}

Results: There was no difference in airway leak pressure between the two devices $\left(24.4 \pm 4.0\right.$ vs $23.6 \pm 3.8 \mathrm{~cm} \mathrm{H}_{2} \mathrm{O}$ $p>0.05)$. Forty-one (91\%) LMA Supremes and forty $(88.8 \%)$ i-gels were successfully inserted on the first attempt, with similar ease, and comparable times $(13.8 \pm 4.9 \mathrm{sec}$ for LMA Supreme vs $14.5 \pm 6.7$ sec for i-gel; $p>0.05$ ). Gastric tube insertion was easier and achieved more quickly with LMA Supreme compared to i-gel $(9.0 \pm 3.1 \mathrm{sec} v s 14.3 \pm 7.5 \mathrm{sec}$, respectively; $p<0.01)$, but clinical significance of this finding is questionable. There was blood on removal of four LMA Supremes and three i-gel. Four patients in the LMA Supreme group and one patient in the i-gel group experience mild postoperative sore throat.

Conclusion: Both LMA Supreme and i-gel are equally effective ventilator devices for diagnostic gynecological laparoscopic procedures.

Keywords: Supraglottic airway device, Laparoscopy surgery, LMA supreme, i-gel.

How to cite this article: Chattopadhyay S, Goswami S. A Comparative Study of Two Disposable Supraglottic Devices in Diagnostic Laparoscopy in Gynecology. J South Asian Feder Obst Gynae 2013;5(3):124-128.

Source of support: Nil

Conflict of interest: None declared

\section{INTRODUCTION}

Stimulation of oropharyngolaryngeal structures is an important factor in the hemodynamic stress response associated with tracheal intubation, ${ }^{1}$ and may lead to left ventricular failure, myocardial ischemia in the presence of coronary artery disease or hypertension. ${ }^{2}$ All laparoscopic procedures including gynecological laparoscopic day care procedures are known to produce increase in pressor response. ${ }^{3}$

Attempts to prevent the pressor response to laryngoscopy and intubation are being made by using alternative guiding devices such as laryngeal mask airway ${ }^{4}$ (LMA), with which the incidence of pressor response may be reduced. ${ }^{5}$ Supraglottic airway devices with gastric access tubes like the Proseal LMA (PLMA) are increasingly being used in surgery requiring general anesthesia and positive pressure ventilation. ${ }^{6}$ However, these devices are costly as they are autoclavable.

Till recently, the disposable variety of LMA (LMA Unique) did not have any gastric access channel or bite block. However, the LMA Supreme ${ }^{\mathrm{TM}}$ (Laryngeal Mask Company, United Kingdom) is a newly introduced disposable LMA with an integral bite block, inflatable cuff and a separate gastric access tube. ${ }^{7}$ Recently, Verghese et al published a crossover trial with 36 female patients showing equal performance of the LMA Supreme and the PLMA. ${ }^{8}$

The i-gel ${ }^{\mathrm{TM}}$ (Intersurgical Ltd, United Kingdom), is another recently developed single-use supraglottic airway device which also features an additional tube to introduce a gastric suction catheter along with an in-built bite block. Its unique design does not need an inflatable cuff because the thermoplastic elastomer provides the seal. ${ }^{9}$ It is a reasonable alternative to tracheal intubation during pressure-controlled ventilation, and is easy to insert while providing an adequate seal. ${ }^{10}$ LMA Supreme differs from the i-gel in that it is constructed of medical grade silicone, and has an inflatable cuff, a reinforced tip, and an anatomically pre-shaped semi-rigid airway tube.

In the current study we aimed to compare the efficacy of the noninflatable cuff of the i-gel with the inflatable cuff of the LMA Supreme in providing an adequate seal for gynecological laparoscopic surgery in the Trendelenburg position in paralyzed patients receiving controlled ventilation. We compared the ease of insertion, hemodynamic response and time to insertion, efficacy in spontaneous ventilation and complications associated with these two disposable supraglottic airway devices of similar sizes (size 3).

\section{MATERIALS AND METHODS}

After obtaining approval from the Institutional Ethics Committee we performed this study from April to December 2011 in the Department of Obstetrics and Gynecology, Medical College Kolkata on 90 consenting women of American Society of Anesthesiologists (ASA) physical status I and II, scheduled for elective diagnostic gynecological laparoscopy. We selected patients in the age group 18 to 60 years and weight between 30 and $50 \mathrm{~kg}$, and excluded patients with a predicted difficult airway, a high risk of regurgitation or aspiration, cardiorespiratory problems, bleeding disorders, preoperative sore throat, any past history of abdominal operations or an estimated operation time $>1$ hour.

Patients were randomly allocated into two groups, 'LMA Supreme' or 'i-gel', using a computer-generated random number table. After recruitment, the enrolling investigators 
opened the sealed opaque envelopes that concealed the group allocation. Participants were blinded to their group allocation. The size of the airway was chosen in accordance to manufacturers' recommendations based on weight (30 to 50 $\mathrm{kg}$ for this study) and we chose size 3 of both devices as per the recommendation of the manufacturers.

All the patients were premedicated 2 hours before operation with tablet Midazolam $7.5 \mathrm{mg}$ and a capsule containing a combined preparation of Omeprazole $20 \mathrm{mg}$ and Domperidone $30 \mathrm{mg}$. Patients were placed supine with the head in a neutral position and standard monitoring was instituted before induction of anesthesia, i.e. pulse oximetry, electrocardiograph and noninvasive blood pressure. Pre-oxygenation was carried out with high flow oxygen for 3 minutes before induction of anesthesia with intravenous fentanyl $1.5 \mu \mathrm{g} / \mathrm{kg}$ and propofol $2.5 \mathrm{mg} / \mathrm{kg}$. Thereafter sevoflurane was started at $8 \%$ dial concentration and either lubricated i-gel or LMA Supreme was inserted after the jaw were sufficiently slackened as per standard insertion technique recommended by the manufacturers. ${ }^{11,12}$

All insertions were performed by senior anesthesists who had performed at least 10 LMA Supreme and i-gel insertions before the commencement of the trial. Insertion difficulty was graded 1 (easy) to 5 (impossible) by the investigator. ${ }^{13}$ The cuff of the LMA Supreme was inflated with air to attain a cuff pressure of $60 \mathrm{~cm} \mathrm{H}_{2} \mathrm{O}$ as measured with a handheld aneroid pressure gauge. A side stream capnograph was connected for end-tidal carbon-dioxide $\left(\mathrm{EtCO}_{2}\right)$ readings. Patients were made to ventilate spontaneously with an inspired concentration of sevoflurane of 2 to $2.5 \%$ to maintain an $\mathrm{EtCO}_{2}$ between 35 and $45 \mathrm{~mm} \mathrm{Hg}$ and a saturation of $95 \%$ or above, with assisted manual ventilation as and when required. The appearance of at least six consecutive $\mathrm{EtCO}_{2}$ traces denoted establishment of effective ventilation. Otherwise, the device was completely removed for another insertion attempt. Three insertion attempts were allowed. Each 'attempt' was defined as re-insertion of the airway device into the mouth. We defined 'insertion failure' of the device as one comprising $>3$ unsuccessful attempts or if the entire process of insertion exceeded 120 seconds. It was planned that in case of failure of either device, the airway will be secured according to the decision of the attending anesthetist and the case will be excluded from the study.

A size 10 FG gastric tube was inserted through the gastric drain outlet of both these devices. These gastric tubes were pre-lubricated with a water-soluble lubricant. Ease of insertion was graded 1-3 ( 1 = easy, 2 = difficult, 3 = impossible). Other variables noted were time to insert and confirmation of placement by detection of injected air by auscultation of the epigastrium and aspiration of gastric contents.

Airway leak pressure was measured after closing the adjustable pressure limiting valve with a fresh gas flow of $3 \mathrm{~L} /$ minutes, noting the airway pressure at equilibrium or when there was an audible air leak from the throat. ${ }^{14}$ The epigastrium was also auscultated when measuring the oropharyngeal leak pressure to detect any air entrainment in the stomach. The maximum pressure allowed was $30 \mathrm{~cm} \mathrm{H}_{2} \mathrm{O}$. We also observed for blood pressure and heart rate every minute for the first
5 minutes from induction of anesthesia, and any maneuvers that were required to optimize positioning or ventilation.

All patients were placed in Trendelenburg position after the surgeon created a pneumoperitoneum using carbon dioxide insufflation and inserted the laparoscopic ports. The magnitude of this Trendelenburg position was kept at $30^{\circ}$. Port insertion attempts and duration of laparoscopy were noted in all cases. During the whole period of pneumoperitoneum intra abdominal pressure and airway pressure were recorded every minute in both the groups.

At end of surgery sevoflurane was turned off and supraglottic airway device removed when there was spontaneous eye opening of patient on command. We observed for any visible blood on the device. Other parameters observed were airway insertion times, ventilatory parameters and complications of placement (desaturation $<95 \%$, gross regurgitation or aspiration (defined as fluid in the ventilation tube), bronchospasm, mucosal, lip, tongue or dental injury and postoperative sore throat (assessed at 45 minutes after removal of the device). These were done by an un-blinded observer who was not involved in the study.

Sample size was based on a pilot study we conducted involving 20 LMA Supreme insertions that demonstrated a mean (SD) oropharyngeal leak pressure of $23 \mathrm{~cm} \mathrm{H}_{2} \mathrm{O}$. To detect a difference of $10 \%$, prospective power analysis at $80 \%$ power and the 0.05 level of significance (two-tailed) showed that a sample size of 43 patients would be required. Therefore, we recruited at least 47 patients per group to account for dropouts and protocol breaches. We used Student's t-test to analyze insertion times and other continuous data, the Mann-Whitney U-test for noncontinuous data, Fisher's exact test for comparison of side effects. All statistical analyses were performed using SPSS $13.0^{\mathrm{TM}}$ (SPSS Inc., Chicago, IL, USA) software. A p-value of $<0.05$ was deemed statistically significant.

\section{RESULTS}

We initially recruited 48 cases in the LMA group and 47 in the i-gel group. In three case, in the LMA group and two cases in the i-gel group the laparoscopic findings necessitated further procedures and thus were excluded from the study. That left us with a total of 90 cases (45 cases in each group) and all the patients recruited completed the present study. Their demographic characteristics and operative characteristics are outlined in Table 1. First time insertion occurred in 41 patients (91.1\%) of LMA Supreme group and $40(88.8 \%)$ patients of

Table 1: Demographic and operative characteristics

\begin{tabular}{lccc}
\hline & $\begin{array}{c}\text { LMA Supreme } \\
(n=45)\end{array}$ & $\begin{array}{c}i \text {-gel } \\
(n=45)\end{array}$ & $p$-value \\
\hline Age (years) & $27.4 \pm 8.6$ & $28.1 \pm 7.9$ & $>0.05$ \\
Weight $(\mathrm{kg})$ & $42.6 \pm 5.3$ & $43 \pm 5.1$ & $>0.05$ \\
Height $(\mathrm{cm})$ & $140 \pm 7.7$ & $142.1 \pm 6.8$ & $>0.05$ \\
ASA grade (I/II) & $34 / 11$ & $33 / 12$ & $>0.05$ \\
Duration of surgery (min) & $15.4 \pm 6.7$ & $16.1 \pm 6.1$ & $>0.05$ \\
Duration of anesthesia (min) & $20.4 \pm 3.6$ & $21.1 \pm 4.1$ & $>0.05$ \\
\hline
\end{tabular}


Table 2: Comparison of insertion characteristics and effects of the supraglottic devices

\begin{tabular}{|c|c|c|c|}
\hline & $\begin{array}{l}\text { LMA supreme } \\
\quad(n=45)\end{array}$ & $\begin{array}{c}i-g e l \\
(n=45)\end{array}$ & $p$-value \\
\hline Size 3 airway & 45 & 45 & - \\
\hline First attempt success rate, $\mathrm{n}(\%)$ & $41(91.1 \%)$ & $40(88.8 \%)$ & $>0.05$ \\
\hline Second and third attempt success rate, $\mathrm{n}(\%)$ & $4(8.8 \%)$ & $5(11.1 \%)$ & $>0.05$ \\
\hline \multicolumn{4}{|l|}{ Ease of insertion (grade 1 to 5 ) } \\
\hline Grade $1=$ easy & 41 & 41 & $>0.05$ \\
\hline Grade 2 = not so easy & 4 & 4 & $>0.05$ \\
\hline Grade 3 to $5=$ difficult, very difficult and impossible & 0 & 0 & - \\
\hline Time for airway insertion (sec) & $13.8 \pm 4.9$ & $14.5 \pm 6.7$ & $>0.05$ \\
\hline \multicolumn{4}{|l|}{ Ease of gastric tube (10FG) insertion } \\
\hline Grade $1=$ easy & $45(100 \%)$ & $35(77.7 \%)$ & $<0.001^{*}$ \\
\hline Grade 2 = difficult & 0 & $10(22.2 \%)$ & $<0.001^{*}$ \\
\hline Grade $3=$ impossible & 0 & 0 & \\
\hline Time for gastric tube insertion (sec) & $9 \pm 3.1$ & $14.3 \pm 7.5$ & $<0.01^{*}$ \\
\hline Airway leak pressure $\left(\mathrm{cm} \mathrm{H}_{2} \mathrm{O}\right)$ & $24.4 \pm 4$ & $23.6 \pm 3.8$ & $>0.05$ \\
\hline Airway pressure $<20 \mathrm{~cm} \mathrm{H}_{2} \mathrm{O}, \mathrm{n}(\%)$ & $3(6.6 \%)$ & $6(13.3 \%)$ & 0.5 \\
\hline Visible blood at time of removal, $n(\%)$ & $4(8.8 \%)$ & $3(6.6 \%)$ & $>0.05$ \\
\hline Lip trauma, n (\%) & $2(4.4 \%)$ & $3(6.6 \%)$ & $>0.05$ \\
\hline Incidence of postoperative sore throat, n (\%) & $4(8.8 \%)$ & $1(2.2 \%)$ & $<0.05^{*}$ \\
\hline
\end{tabular}

${ }^{*}$ p-value of 0.05

the i-gel group $(\mathrm{p}>0.05)$, while the rest were inserted during the second attempt. None was rated as difficult to insert. The mean times from insertion of the airway device to the first capnograph trace were similar for both LMA Supreme and i-gel $(13.8 \pm 4.9$ vs $14.5 \pm 6.7 \mathrm{sec} ; \mathrm{p}>0.05)$. However, it was more difficult and took significantly longer to insert the gastric tube in the i-gel group $(14.3 \pm 7.5$ vs $9.0 \pm 3.1 \mathrm{sec} ; \mathrm{p}<0.01)$. More importantly the airway leak pressure between these two devices was similar $\left(24.4 \pm 4.0\right.$ vs $23.6 \pm 3.8 \mathrm{~cm} \mathrm{H}_{2} \mathrm{O}$; $\mathrm{p}>0.05)$. On removal, visible blood indicative of mucosal injury was noticed on three i-gel and four LMA Supreme, which was comparable. Minor lip trauma occurred upon insertion in three patients with i-gel and in two patients with LMASupreme. Four patients in the LMA Supreme group and one patient in the i-gel group experienced mild postoperative sore throat (Table 2).

No patient in the study experienced regurgitation, bronchospasm, tongue trauma, or dental injury. The hemodynamic consequences of airway placement did not differ significantly between the groups, this is not shown in the table.

Table 3 reflects the operative parameters. There was no significant difference in mean number of port insertion attempts, the duration of laparoscopy, mean intra-abdominal pressure and mean airway pressure in between the two groups.

\section{DISCUSSION}

In our study, both the LMA Supreme and i-gel were easy and quick to insert, with an equally high successful insertion rate on the first attempt. However, insertion success for both devices was less than reported previously with a first success rate of $97 \%$ for the i-gel $^{15}$ and $100 \%$ easy insertion for the LMASupreme. ${ }^{12}$ Both airway devices achieved comparable oropharyngeal leak pressures and proved to be effective ventilatory devices for gynecological laparoscopic procedures, similar to other studies. ${ }^{13,16}$ All gastric tubes were successfully inserted on the first attempt. It was, however, more difficult to insert a well-lubricated 10-FG gastric tube into the i-gel due to the smaller aperture of the gastric access port, and therefore this took longer time. This was similar to the studies of Teoh et $\mathrm{al}^{16}$ and Fernandez et al. ${ }^{17}$ We measured oropharyngeal leak pressure using two methods: auscultation and manometer stability. ${ }^{14}$ The values obtained have been found to be similar using either method. There was no air leak into the stomach or gastric insufflations in any of our patients at the equilibrium leak pressure. ${ }^{13,16}$

Uppal et $\mathrm{al}^{10}$ showed that the i-gel had no significant difference in gas leak compared with tracheal tubes when ventilating at moderate pressures up to 15 to $20 \mathrm{~cm} \mathrm{H}_{2} \mathrm{O}$, but did not study pressures higher than $25 \mathrm{~cm} \mathrm{H}_{2} \mathrm{O}$. All our patients were placed in the head-down position as required in most gynecological laparoscopic procedures lasting approximately 15 minutes. However, we found the mean airway pressures in our study did not exceed $25 \mathrm{~cm} \mathrm{H}_{2} \mathrm{O}$ despite the Trendelenburg position and pneumoperitoneum. This could be due to the relatively low BMI in our female population and our surgeons' care in limiting intra-abdominal pressures throughout the operation (within $15 \mathrm{~mm} \mathrm{Hg}$ ).

Theiler et $\mathrm{al}^{13}$ recently compared the use of both the LMA Supreme and the i-gel in a randomized, crossover setting in simulated difficult airways and found equal insertion success rates (95\% for the LMA Supreme vs $93 \%$ for the i-gel). In our study, supraglottic devices could be inserted in all our patients within two attempts and were not difficult to insert, these were comparable to the study of Teoh et al. ${ }^{16}$

\begin{tabular}{|c|c|c|c|}
\hline & $\begin{array}{l}\text { LMA supreme } \\
\quad(n=45)\end{array}$ & $\begin{array}{c}i-g e l \\
(n=45)\end{array}$ & $p$-value \\
\hline Port insertion attempts (mean) & 1.15 & 1.08 & $>0.05$ \\
\hline Duration of laparoscopy (min) & $10.4 \pm 3.2$ & $10.8 \pm 3.4$ & $>0.05$ \\
\hline $\begin{array}{l}\text { Average intra-abdominal } \\
\text { pressure }(\mathrm{mm} \mathrm{Hg})\end{array}$ & $15.5 \pm 3.7$ & $15.2 \pm 3.6$ & $>0.05$ \\
\hline $\begin{array}{l}\text { Mean airway pressure after } \\
\text { pneumoperitoneum }(\mathrm{mm} \mathrm{Hg})\end{array}$ & $23.4 \pm 4.8$ & $22.5 \pm 5$ & $>0.05$ \\
\hline
\end{tabular}


Fernandez et $\mathrm{al}^{17}$ evaluated the ease of insertion of the LMA Supreme and the i-gel in 85 patients requiring mechanical ventilation, they found the i-gel had longer insertion times (LMA Supreme $27.1 \mathrm{sec} v s$ i-gel $32.5 \mathrm{sec}$ ) and lower success on first attempt $(97.6 \%$ vs $85.7 \%)$. These values were much higher than our study findings, and may reflect upon the fact that we allowed only senior anesthesiologists with a number of previous experiences with these devices to perform the airway insertions. However, as in our study, they found both devices provided an effective seal and efficacy of ventilation with similar minor incidences of complications.

We found oropharyngeal leak pressures of $<20 \mathrm{~cm} \mathrm{H}_{2} \mathrm{O}$ in three LMA Supreme and six i-gel cases after uneventful insertions. Each case had gastric tubes successfully inserted and no audible throat noise or leak was heard at the airway pressure when equilibrium was achieved on the manometer. Ventilation was not compromised in any of these cases, with delivery of adequate tidal volumes and anesthetic agents for the duration of surgery. This finding challenges the widely perceived notion that a leak pressure of $<20 \mathrm{~cm} \mathrm{H} \mathrm{H}_{2} \mathrm{O}$ in supraglottic airway devices necessitates removal of the 'malpositioned' device due to potential to compromise its ventilatory efficacy. Indeed, a recent randomized crossover study that compared the disposable LMA Supreme with the Proseal concluded that ventilation was not affected despite lower leak pressures. ${ }^{18}$

The i-gel was designed to create a perilaryngeal anatomical seal without an inflatable cuff, decreasing the risk of compression trauma in cadaveric studies. ${ }^{9}$ The use of the i-gel has now been shown clinically to result in fewer postoperative sore throat and neck complaints compared with disposable LMAs. ${ }^{19}$ Blood staining on removal was found on three i-gel $(6.6 \%)$ and four $(8.8 \%)$ LMA Supreme devices. Four patients $(8.8 \%)$ with the LMA Supreme and one patient $(2.2 \%)$ with i-gel experienced mild postoperative sore throat. Paradoxically, patients' tongues can get caught inside the i-gel's bowl during insertion and any undue resistance encountered or force used on insertion can result in tongue trauma, although this is quite rare, reported in 3 out of $1100 \mathrm{i}$-gel insertions. ${ }^{20} \mathrm{We}$ did not encounter any of these in our study. Our study has a few limitations. We did not measure the leak pressure at the end of surgery for either device. It could have perhaps added important information as reports have emerged that the seal of the i-gel seems to improve over time due to the thermoplastic cuff's warming to body temperature. ${ }^{12} \mathrm{We}$ did not use fiberoptic bronchoscopy (FOB) to assess the anatomical position of the i-gel and LMA Supreme in relation to the vocal cords for two reasons. First, we wanted this study to reflect our clinical practice and replicate our daily workflow and high surgical turnover. ${ }^{16}$ It was deemed not clinically and logistically feasible to perform FOB in all cases. Second, there is evidence that anatomical findings do not necessarily correlate with clinical consequences. $^{21}$ It is also impossible to blind the airway operator to the device used, hence there is a potential for bias. We only studied non-obese women and the results cannot directly be extrapolated to other types of patients. ${ }^{16}$

\section{CONCLUSION}

The airway seal pressure offered by the i-gel is comparable to that of the LMA Supreme. It took a longer time to insert the gastric tube in the i-gel while more post operative sore throat occurred with LMA Supreme, even though the clinical relevance is questionable. Both supraglottic airway devices are comparable in terms of ease of insertion, success rates on the first attempt, time of insertion as well as oropharyngeal leak pressure, proving to be equally effective ventilator devices for gynecological laparoscopic procedures in our study. Most of the diagnostic laparoscopy in gynecology are office procedures of short duration and therefore the supraglottic devices which can avert the complications of endotracheal intubation are feasible emerging alternative options.

\section{REFERENCES}

1. Shribman AJ, Smith G, Achola KH. Cardiovascular and catecholamine responses to laryngoscopy with and without tracheal intubation. Br J Anaesth 1987;59:295-299.

2. Association of Anaesthetists of Great Britain and Ireland. Recommendations for Standards of Monitoring During Anaesthesia and Recovery, 4th ed. London: AAGBI, 2007.

3. Ahonen J, Jokela R, Uutela K, Huiku M. Surgical stress index reflects surgical stress in gynaecological laparoscopic day care surgery. Br J Anaesth 2007;98:456-461.

4. Kaul TK, Valecha V, Kathuria S, Chatrath R, Gautam PL. Catecholamine responses to endotracheal intubation and laryngeal mask airway insertion. Ind J Anaesth 1999;43:30-35.

5. Brodrick PH, Webster NR, Nunn JE. The laryngeal mask airway: a study of 100 patients during spontaneous breathing. Anaesthesia 1989;44:238-241.

6. Cook TM, Lee G, Nolan JP. The ProSeal ${ }^{\mathrm{TM}}$ laryngeal mask airway: a review of the literature. Canadian Journal of Anesthesia 2005;52:739-760.

7. Timmermann A, Cremer S, Eich C, Kazmaier S, Bräuer A, Graf BM, Russo SG. Prospective Clinical and Fiberoptic Evaluation of the Supreme Laryngeal Mask Airway ${ }^{\mathrm{TM}}$. Anesthesiology 2009;110:262-265.

8. Verghese C, Ramaswamy B. LMA-Supreme: a new single-use LMA with gastric access: A report on its clinical efficacy. Br J Anaesth 2008;101:405-410.

9. Levitan RM, Kinkle WC. Initial anatomic investigations of the i-gel airway: a novel supraglottic airway without inflatable cuff. Anaesthesia 2005;60:1022-1026.

10. Uppal V, Fletcher G, Kinsella J. Comparison of the i-gel with the cuffed tracheal tube during pressure-controlled ventilation. Br J Anaesth 2009;102:264-268.

11. Intersurgical. i-Gel Users Guide. Available from http:// www.intersurgical. com/utils/GetFile.aspx? FileID $=29456$ (accessed June 2010).

12. van Zundert A, Brimacombe J. The LMA Supreme-a pilot study. Anaesthesia 2008;63:209-210.

13. Theiler LG, Kleine-Brueggeney M, Kaiser D, et al. Crossover comparison of the laryngeal mask supreme and the i-gel in simulated difficult airway scenario in anesthetized patients. Anesthesiology 2009;111:55-62.

14. Keller C, Brimacombe JR, Keller K, Morris R. Comparison of four methods for assessing airway sealing pressure with the laryngeal mask airway in adult patients. Br J Anaesth 1999; 82:286-267. 
15. Richez B, Saltel L, Banchereau F, Torrielli R, Cros AM. A new single use supraglottic airway device with a noninflatable cuff and an esophageal vent: an observational study of the i-gel. Anesth Analg 2008;106:1137-1139.

16. Teoh WHL, Lee KM, Suhitharan T, Yahaya Z, Teo MM, Sia ATH. Comparison of LMA Supreme vs the i-gel ${ }^{\mathrm{TM}}$ in paralysed patients undergoing gynaecological laparoscopic surgery with controlled ventilation. Anaesthesia 2010;65:1173-1179.

17. Fernandez Diez A, Perez Villafane A, Bermejo Gonzalez JC, Marcos Vidal JM. Supreme laryngeal mask airway vs the i-gel supraglottic airway in patients under general anesthesia and mechanical ventilation with no neuromuscular block: a randomized clinical trial. Revista Espan ola de Anestesiologý'ay Reanimacio'n 2009;56:474-478.

18. Tham HM, Tan SM, Woon KL, Zhao YD. A comparison of the Supreme laryngeal mask airway with the Proseal laryngeal mask airway in anesthetized paralyzed adult patients: a randomized crossover study. Canadian Journal of Anesthesia 2010;57: 672-678.

19. Keijzer C, Buitelaar DR, Efthymiou KM, et al. A comparison of postoperative throat and neck complaints after the use of the i-gel and the La Premiere disposable laryngeal mask: a double- blinded, randomized, controlled trial. Anesth Analg 2009;109: 1092-1095.

20. Michalek P, Donaldson WJ, Hinds JD. Tongue trauma associated with the i-gel supraglottic airway. Anaesthesia 2009;64: 692-693.

21. Van Zundert A, Brimacombe J, Kamphuis R, Haanschoten M. The anatomical position of three extraglottic airway devices in patients with clear airways. Anaesthesia 2006;61:891-895.

\section{ABOUT THE AUTHORS}

\section{Suman Chattopadhyay}

Associate Professor, Department of Anesthesiology, Medical College and Hospital, Kolkata, West Bengal, India

\section{Sebanti Goswami}

Associate Professor, Department of Obstetrics and Gynecology Medical College and Hospital, Kolkata, West Bengal, India

Correspondence Address: 317, New Raipur (Dabur Park), Flat No. 1B, Kolkata-700084, West Bengal, India, Phone: 09831135933 e-mail: sebantigoswami@yahoo.co.in 